\title{
Location-Based Routing Protocol for Energy Efficiency in Wireless Sensor Networks
}

\author{
Hyuntae Cho and Yunju Baek \\ Department of Computer Science and Engineering, Pusan National University, \\ Busan, Republic of Korea \\ marine@juno.cs.pusan.ac.kr, yunju@pusan.ac.kr
}

\begin{abstract}
Energy efficiency in wireless sensor networks is an important design consideration. We present a location-based routing protocol for energy efficiency in wireless sensor networks called GPSR-S(Greedy Perimeter Stateless Routing for wireless Sensor networks). GPSR-S is based on GPSR, which is one of the most well-known location-based routing protocols for wireless ad hoc networks. We improve the energy efficiency of GPSR by considering nodes' energy level and location information. In addition, we modify the address-centric nature of the algorithm into a data-centric one. Simulation results show that GPSR-S performs well in terms of energy efficiency and the number of packets. GPSR-S delivers approximately $10 \%$ fewer packets than GPSR, but the lifetime of the network is $10 \%$ greater.
\end{abstract}

\section{Introduction}

Wireless sensor networks are likely to be widely deployed in the future because they greatly extend the ability to monitor and control the physical environment from remote locations. Such networks are composed of a large number of sensor nodes, which are densely deployed either inside the phenomenon or very close to it. These tiny sensor nodes, which consist of sensing, data processing, and communicating components, use the idea of sensor networks based on the collaborative effort of a large number of nodes. Some of the application areas are health, military, and security.

The position of sensor nodes need not be engineered or pre-determined. This means that sensor network protocols and algorithms must possess self-organizing capabilities. So, wireless sensor networks usually use the protocols and algorithms for wireless ad hoc networks in order to achieve self-organization. Location awareness is another important issue in wireless sensor networks. Most data collection is based on location, so it is desirable that the nodes know their position whenever it is needed. In most cases, location information is needed in order to calculate the distance between two particular nodes so that energy consumption can be estimated. Since there is no addressing scheme (such as IP-addresses) for sensor networks and they are spatially deployed on a region, location information can be utilized in routing data in an energy efficient way.

Although GPSR[3] has been proposed for traditional wireless ad hoc networks, it is not well suited to the unique features and application requirements of sensor 
networks. To illustrate this point, the differences between sensor networks and ad hoc networks are outlined below:

- The number of sensor nodes in a sensor network can be several orders of magnitude higher than the nodes in an ad hoc network.

- Sensor nodes are more densely deployed.

- Sensor nodes are limited in power, computational capacity, and memory.

- Sensor nodes may not have global identification because of the large overhead and large number of sensors.

- Sensor networks are "data-centric" i.e., unlike traditional networks where data is requested from a specific node, data is requested based on particular attributes, such as, "which area has temperature $>50^{\circ} \mathrm{F}$ ?" Data-centric protocols are query-based and depend on the naming of desired data, which helps in eliminating many redundant transmissions.

For these reasons, GPSR should be fixed before it is applied in wireless sensor networks. So, we propose a modified version of GPSR called GPSR-S(GPSR for wireless Sensor networks) to fulfill the requirements of wireless sensor networks. GPSR-S uses energy-aware and geographical information to route a packet towards the target region. Within a region, it uses a forwarding technique to disseminate the packets. Next, we modify wireless sensor networks to be data-centric, where communications are expressed, not in terms of node identifier but in terms of named data.

The remainder of the paper is organized as follow: In Section 2, we discuss related work on wireless sensor networks. In Section 3, we explain location-based routing for energy efficiency in wireless sensor networks. In Section 4, we simulate GPSR-S and compare its performance to GPSR. Section 6 concludes.

\section{Related Work}

Routing protocols in wireless sensor networks are classified into various categories: (i) address-centric and data-centric routing, with respect to method of data acquisition; (ii) flat and hierarchical routing, with respect to the form of the network; and (iii) location-based and location-independent routing protocols.

In data-centric routing, the sink sends queries to certain regions and waits for data from the sensors located in the selected regions. Since data is requested through queries, attribute-based naming is necessary to specify the properties of data. SPIN[6] is the first data-centric protocol, which considers data negotiation between nodes in order to eliminate redundant data and save energy. Later, Directed Diffusion[5] was developed and constituted a breakthrough in data-centric routing. Directed Diffusion is an important milestone in data-centric routing research on sensor networks.

In common with other communication networks, scalability is a major design attribute of sensor networks. A single-tier network can cause the gateway to overload with an increase in sensor density. So, single-gateway architecture is not scalable for a larger set of sensors covering a wider area of interest, because the sensors are typically not capable of long-haul communication. The main aim of hierarchical routing is to efficiently maintain the energy consumption of sensor nodes by involving them in multi-hop communication within a particular cluster and by the sink. Cluster forma- 
tion is typically based on the energy reserve of sensors and sensor's proximity to the cluster head. LEACH(Low-Energy Adaptive Clustering Hierarchy)[7] is one of the first hierarchical routing approaches for sensor networks. The idea proposed in LEACH has been an inspiration for many hierarchical routing protocols such as TEEN(Threshold sensitive Energy Efficient sensor Network protocols)[7], and APTEEN(Adaptive Periodic TEEN)[8].

Most of the running protocols for sensor networks require location information for sensor nodes. Since there is no addressing scheme for sensor networks and they are deployed spatially across a region, location information can be utilized in routing data in an energy-efficient way. For instance, if the region to be sensed is known, using the location of sensors, the query can be diffused only to that particular region, which will reduce the number of transmissions significantly. GPSR[3] is one of the most well known geographic routing algorithms in wireless ad hoc networks. Other hierarchical schemes in use are GEAR (Geographical and Energy Aware Routing)[9], and LAR (Location Aided Routing)[10].

GPSR uses the position of nodes and a packet's destination to make decisions about packet forwarding. GPSR makes greedy forwarding decisions, using only information about a node's immediate neighbors in the network topology. When a packet reaches a region where greedy forwarding is impossible, the algorithm recovers by routing around the perimeter of the region. By keeping state only about the local topology, GPSR scales better in per-node state than shortest-path and ad-hoc routing protocols as the number of network destinations increases. Under mobility's frequent topology changes, GPSR can use local topology information to find correct new routes quickly. But, when GPSR is used in wireless sensor networks, it has some shortcomings. Because it uses greedy forwarding to distance from the node to the destination, the specific nodes which located on the optimum path can only consume energy. Also, it reduces energy efficiency due to address-centric routing. In order to fulfill the above requirements, in the next section we propose a location-based routing protocol for energy efficiency.

\section{Location-Based Routing Protocol for Energy Efficiency in Wireless Sensor Networks}

Wireless sensor networks are composed of a number of nodes that have limited energy resources. The energy depletion of nodes in wireless sensor networks brings about the partitioning of the network. To avoid such partitioning, the consumption $f$ energy in wireless networks must be distributed fairly across all nodes. Furthermore, the main aim at the network layer is to find ways for energy-efficient route setup and reliable relaying of data from the sensor nodes to the sink so that the lifetime of the network is maximized. So, the forwarding process that we propose consists of two phases: (1) forwarding node selection phase, and (2) data dissemination inside the target region.

\subsection{Forwarding Node Selection Technique}

Packets are marked by their originator with their destinations' locations. Upon receiving a packet, a node checks its neighbors to see if there is one that is closer to 
the destination. If such a neighbor exists, GPSR-S picks a next-hop node among all neighbors that are closer to the destination than itself. Here, each node knows its own location and remaining energy level, and its neighbors' location and remaining energy levels through a simple "neighbor hello" protocol. To select a closer neighbor to the destination, GPSR-S uses information about nodes' energy level and location.

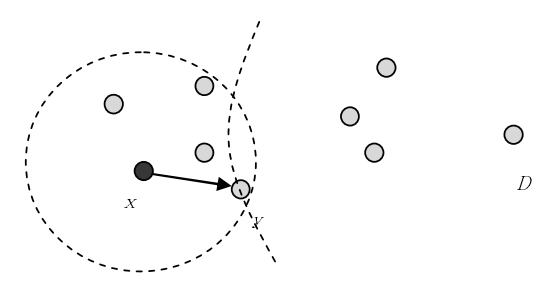

Fig. 1. Forwarding node selection

A formula proposed for the next forwarding node selection is as follows:

next hop $=\max \left(\alpha \frac{\operatorname{dist}(N, D)-\operatorname{dist}(N i, D)}{r}+(1-\alpha) \frac{\operatorname{Er}(N i)}{\operatorname{Ef}(N i)}\right)$ [formula 1]

Where $N i$ is the neighbor node of node $N, D$ indicates the centroid of the target region, and $\operatorname{dist}(x, y)$ is the distance between node $x$ and node $y . r$ is the initial radio transmission coverage, $\operatorname{Er}(\mathrm{Ni})$ is the remaining energy level of node $\mathrm{Ni}$, and $E f(\mathrm{Ni})$ is the initial energy level of all nodes. In formula $1, \alpha$ can be adjusted to emphasize either the minimizing path length to the destination or the balancing energy consumption. $\alpha$ has a value between 0 and 1 . Note that each node can obtain its location information, energy level, and its neighbor's information, which presumably are already available due to the needs of sensor network applications through hello messages. After selecting a forwarding node, the forwarding node selection process repeats until the packet reaches the target region.

The power of this forwarding to route using only neighbor nodes' positions come with one attendant drawback: there are topologies in which the only route to a destination requires that a packet move temporarily farther in geometric distance from the destination. We address the failure of pure greedy routing to find paths in the presence of voids, by introducing perimeter traversal algorithms(used in GPSR) for forwarding packets around voids.

After describing the right-hand rule for traversing a graph, GPSR characterizes the behavior of the right-hand rule on wireless network graphs, and observes the role played by crossing edges in the graph in interfering with the right-hand rule traversal. GPSR then introduces perimeter probing while employing a no-crossing heuristic to eliminate crossing edges from the graph, and uses the resulting state to forward around voids. 


\subsection{Data Dissemination Within Destination Region}

As mentioned above, the wireless sensor networks use data-centric routing instead of address-centric routing. GPSR sends queries to one node instead of multiple nodes. For the description of the algorithm, we assume a rectangular region specification. The form of query sent by sink is below.

$$
\begin{aligned}
& \text { type }=\text { four-legged animal } \\
& \text { rect }=[100,200,200,400] \\
& \text { etc... }
\end{aligned}
$$

Each query packet has a target region specified in some way. The centroid of the target region can be calculated as follows:

$$
\text { Centroid: }(D x, D y)=[(D x 1+D x 2) / 2,(D y 1+D y 2) / 2] \quad \text { [formula 2] }
$$

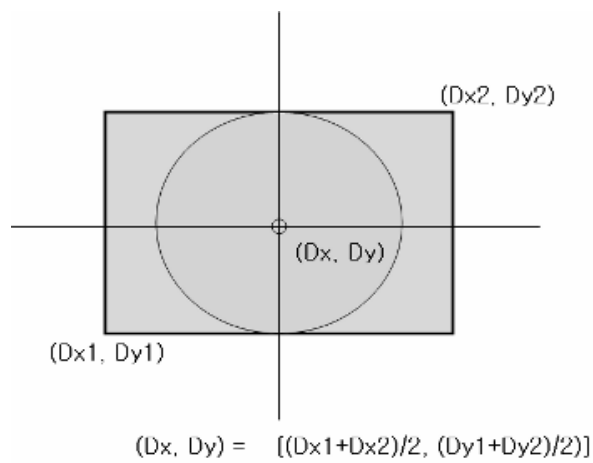

Fig. 2. The centroid of the destination region
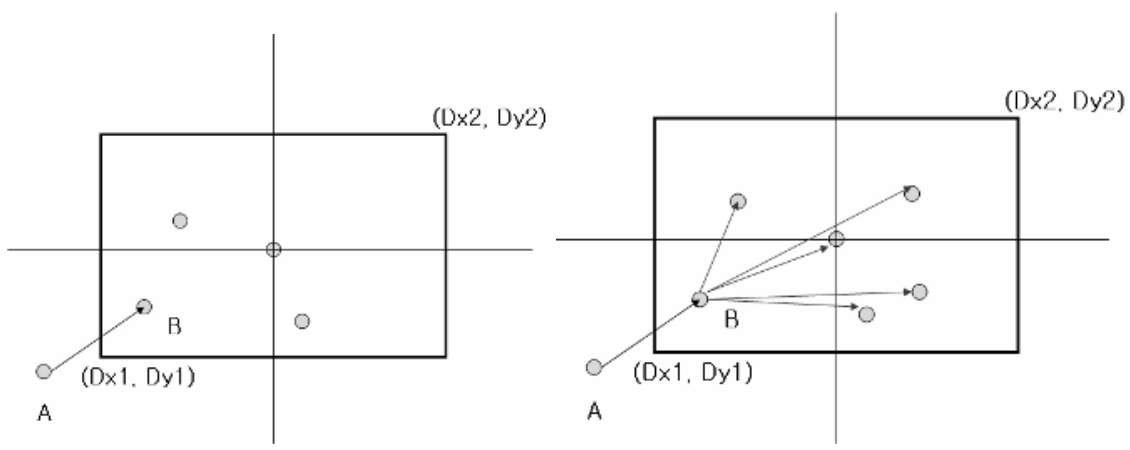

Fig. 3. Data dissemination inside the target region

As shown in Figure 3, after the first packet reaches the destination, B receives a packet from A, and finds itself inside the target region. If the current node is the only one inside this region, the packet is forwarded within this region. Once the packet is 
inside the target region, a simple flooding with duplicate suppression scheme can be used to flood the packet inside the target region.

However, flooding is expensive in terms of energy consumption, due to the fact that in this simple flooding scheme, every node has to broadcast once and all its neighbors receive this broadcast message. This is especially expensive in high-density networks. Therefore, we can use a RGF(Recursive Geographic Forwarding) approach to disseminate the packet inside the target region[9].

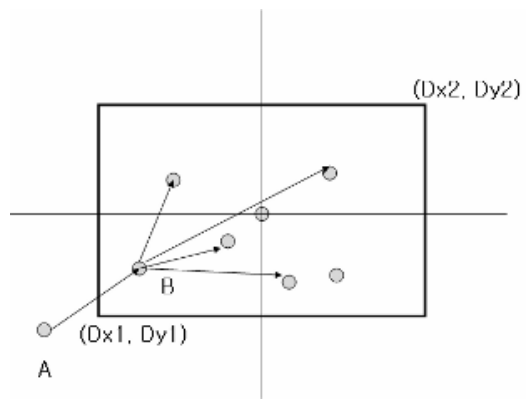

Fig. 4. Recursive Geographic Forwarding

RGF supposes that the target region is the big rectangle, and now node receives a packet for a region, and finds itself inside this region. In this case, node B creates four new copies of a packet bound to 4 subregions(as shown by the four small rectangles in figure 4). This recursive splitting and forwarding procedure is repeated until the stop condition is satisfied.

\section{Simulation Results}

We study and compare GPSR with GPSR-S. In addition, we evaluate how our algorithm compares with GPSR. Since the original GPSR does not handle routing to a region, it does not consider energy efficiency. We have augmented GPSR to route packets to a region, thus considering energy efficiency. The preformance measure is increased network lifetime due to routing in a target region and energy awareness.

To evaluate performance, we used NS-2[12]. The test model is MICA2 mote, designed by UC Berkeley. It operates at $433 \mathrm{MHz}$ radio frequency, and consumes from $5.3 \mathrm{~mA}$ to $26.7 \mathrm{~mA}$ in Tx mode and $7.4 \mathrm{~mA}$ in Rx mode. The antenna is $10 \mathrm{~cm}$ high, it is omni-directional, and its transmission range is $10 \mathrm{~m}$.

In the study reported in this paper, we varied network size, density and other parameters. More specifically, the simulation results shown in this section include networks ranging in size from 30 to 150 . For network simulation, its geometric area was $50 \times 50$ square. The initial location of each node was randomly generated. The 802.11 was used in the radio model. The initial data size was 42 bytes and the transmission period was between 0.5 and $2 \mathrm{sec}$. The simulations were run for 100 
seconds, the speed of nodes was set to $1 \mathrm{~m} / \mathrm{sec}$, and the initial energy of each node was $10 \mathrm{~J}$.

Figure 5 shows the number of live nodes up to 20. This graph considers only energy efficiency. That is, it uses only a forwarding node selection method. This result shows that when 100 seconds had elapsed, 52 nodes in GPSR, 72 nodes in GPSR$\mathrm{S}(\alpha=0.3)$, and 75 nodes in GPSR-S $(\alpha=0.4)$ were alive. Intuitively, this means that GPSR-S has a longer network lifetime than GPSR. The reason that GPSR-S prolongs network lifetime compared with GPSR, is that GPSR-S tends to concentrate on greedy forwarding to distance and energy efficiency.

Figure 6 shows the remaining energy distribution of nodes when 2/3 of the entire lifetime has elapsed. Herein, the number of nodes is 90 . As shown in the graph, it is evident that the nodes in GPSR-S consume the energy more fairly than those in GPSR.

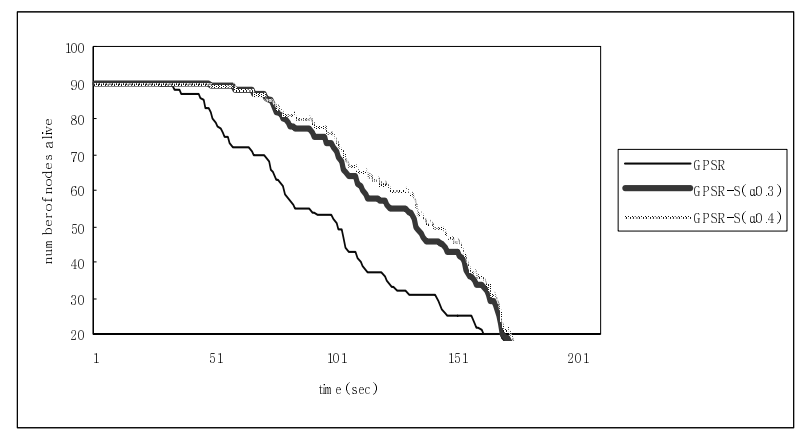

Fig. 5. The number of live nodes

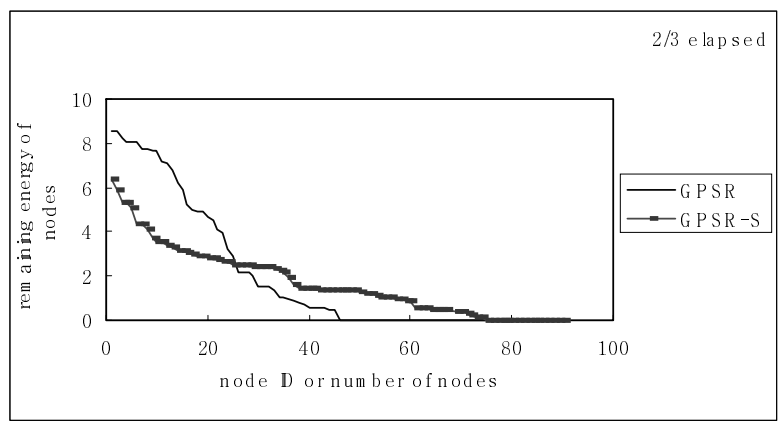

Fig. 6. Energy distribution of nodes within the network

Figure 7 shows the number of packets generated according to $\alpha$. From the graph, it can easily be seen that the number of packets varies according to the value of $\alpha$ and the form of network. Also, a higher $\alpha$ can generate more traffic within the network. 


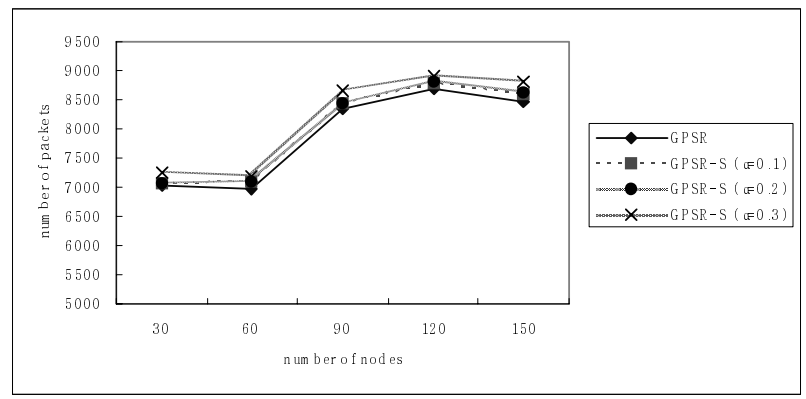

Fig. 7. The number of packets to values of $\alpha$

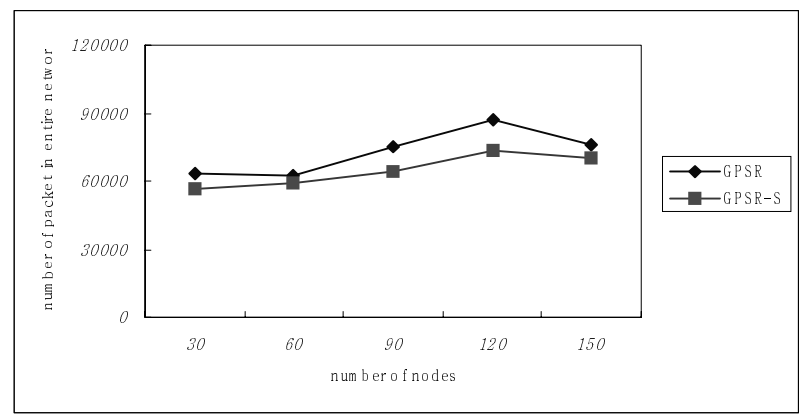

Fig. 8. The number of packets in the entire network

Figure 8 shows the number of packets that are generated in the entire network by applying data-centric routing, which has a target region. There are three nodes in this region. In this case, GPSR-S has $10 \%$ less traffic than GPSR.

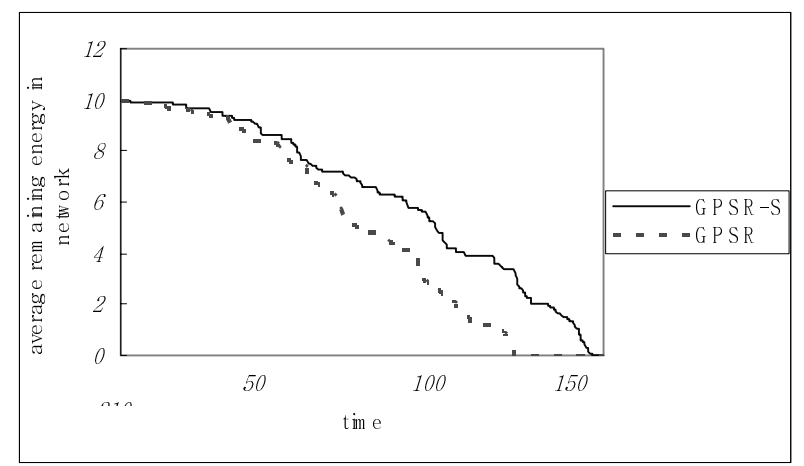

Fig. 9. The average remaining energy of all nodes 
Figure 9 compares GPSR with GPSR-S with respect to energy consumption over time. The performance of GPSR-S is higher with respect to energy consumption and longevity of the network. After 160sec, $1 \%$ of all nodes in GPSR and 3\% of all nodes in GPSR-S remain. It is clear that the amount of energy consumed increases according to the number of packets.

Because our algorithm relies on random decisions, it is important to show that its performance does not vary significantly over several runs.

\section{Conclusion and Future Work}

The traditional routing protocols are not considered to be energy-efficient, so only specific nodes on the optimum path consume energy. We studied the problem of forwarding a packet to nodes in a geographic region of a wireless sensor network. The proposed GPSR-S protocol uses energy-aware and geographic neighbor selection to route a packet towards the target region. Within a region, it uses a forwarding technique to disseminate the packet. These strategies attempt to balance energy consumption and thereby increase network lifetime.

Simulation results show that the GPSR-S consumed energy uniformly while $20 \%$ of the totality of nodes were alive. Data centric routing, moreover, use less traffic than the routing mechanisms which communicate with a specific node. GPSR generates traffic per node as it sends data to nodes. In GPSR-S, about $10 \%$ can be reduced in traffic by adapting data-centric routing. However, there is a possibility of delay in data transmission. GPSR-S, which is proposed in this paper, results in a better protocol for wireless sensor networks because it reflects the energy efficiency and datacentric routing appropriately. This strategy attempts to balance energy consumption and thereby increase network lifetime. We evaluated the performance through simulation. In further work, we intend to investigate how real implementation affects the performance of the protocol.

Acknowledgement. This work was supported by the Regional Research Centers Program(Research Center for Logistics Information Technology), granted by the Korean Ministry of Education \& Human Resources Development.

\section{References}

[1] I. F. Akyildiz, W. Su, Y. Sankarasubramaniam, E. Cayirci, "Wireless sensor networks: a survey," Computer Networks, vol. 38, pp. 393-422, 2002.

[2] Kemal Akkaya, Mohamed Younis, "A survey on routing protocols for wireless sensor networks," Ad Hoc Networks, pp. 26, 2003.

[3] B. Karp and H.T. Kung. "GPSR: Greedy perimeter stateless routing for wireless networks,” In ACM MOBICOM, Boston, MA, August 2000.

[4] W. Rabiner Heinzelman, A. Chandrakasan, and Hari Balakrishnan. "Energy-Efficient Communication Protocol for Wireless Microsensor Networks," In Proceedings of the 33rd International Conference on System Sciences (HICSS '00), 2000. 
[5] Chalermek Intanagonwiwat, Ramesh Govindan, Deborah Estrin, John Heidemann, Fabio Silva, "Directed diffusion for wireless sensor networking," IEEE/ACM Transactions on Networking (TON), Volume 11, Issue 1, pp : 2 - 16, 2003.

[6] Joanna Kulik, Wendi Rabiner Heinzelman, Hari Balakrishnan, "Negotiation-based Protocols for Disseminating Information in Wireless Sensor Networks," ACM Wireless Networks, 1999

[7] Arati Manjeshwar et al., "TEEN: A Routing Protocol for Enhanced Efficiency in Wireless Sensor Networks," Proc. Second Int'l Workshop Parallel and Distributed Computing Issues in Wireless Networks and Mobile Computing, 2001.

[8] Arati Manjeshwar et al., "APTEEN: A Hybrid Protocol for Efficient Routing and Comprehensive Information Retrieval in Wireless Sensor Networks," IEEE Proc. of the International Parallel and Distributed Processing Symposium(IPDPS'02), Apr. 2002, pp.195202.

[9] Yan Yu, Ramesh Govindan, Deborah Estrin "GEAR : Geographical and Energy Aware Routing : a recursive data dissemination protocol for wireless sensor networks," Technical Report UCLA/CSD-TR -01-0023, UCLA Computer Science Dept., May 2001.

[10] Y.-B. Ko and N. H. Vaidya. "Location-aided routing (LAR) in mobile ad hoc networks," In ACM MOBICOM, October 1998.

[11] http://www.icir.org/bkarp/GPSR/GPSR.html

[12] Network Simulator 2 : http://www.isi.edu/nsnam/ns/

[13] http://www.zigbee.org 